\title{
X-rays from Hot Subdwarfs
}

\author{
Sandro Mereghetti and Nicola La Palombara \\ INAF-IASF Milano, v.Bassini 15, I-20133 Milano, Italy
}

\begin{abstract}
Thanks to the high sensitivity of the instruments on board the XMM-Newton and Chandra satellites, it has become possible to explore the properties of the X-ray emission from hot subdwarfs. The small but growing sample of hot subdwarfs detected in X-rays includes binary systems, in which the Xrays result from wind accretion onto a compact companion (white dwarf or neutron star), as well as isolated sdO stars in which X-rays are probably due to shock instabilities in the wind. X-ray observations of these low mass stars provide information which can be useful also for our understanding of the winds of more luminous and massive early-type stars and can lead to the discovery of particularly interesting binary systems.
\end{abstract}

Keywords: X-rays: stars, binaries; Stars: subdwarfs, mass-loss; Stars: individual: $\mathrm{HD} 49798, \mathrm{BD}+37^{\circ} 442, \mathrm{BD}+37^{\circ} 1977, \mathrm{BD}+28^{\circ} 4211$, $\mathrm{CD}-30^{\circ} 11223$, Feige 34

\section{Introduction}

While soft X-ray emission from massive OB stars has been discovered more than thirty years ago (Seward et al., 1979; Harnden et al., 1979), only in recent years, thanks to the great sensitivity of the X-ray instrumentation carried by the XMM-Newton and Chandra satellites, it has become possible to reveal in the X-ray range also hot stars with much smaller masses and luminosities, such as the hot subdwarfs. These stars have high temperatures, corresponding to $\mathrm{O}$ and $\mathrm{B}$ spectral types, but luminosity values that place them below the main sequence in the HR diagram.

Email address: sandro@iasf-milano.inaf.it, nicola@iasf-milano.inaf.it (Sandro Mereghetti and Nicola La Palombara) 
The B type subdwarfs ( $\mathrm{sdBs}$ ) have masses of $\sim 0.5 M_{\odot}$, effective temperatures $20<T_{\text {eff }}<40 \mathrm{kK}$, and surface gravities $5<\log g<6$. They are interpreted as evolved low-mass stars that lost most of their hydrogen envelopes and are now in the He core burning phase. Subdwarfs of O spectral type (sdOs) constitute a less homogeneous group compared to the sdBs. They show a large range of temperatures $\left(40<T_{\text {eff }}<100 \mathrm{kK}\right)$ and surface gravities $4<\log g<7$, and comprise both He-rich and He-poor stars.

X-ray emission associated with a hot subdwarf was seen for the first time in 1979 (see Sect. 3.1), but it took almost two decades to demonstrate that most of the observed X-rays actually originate from its compact companion star (Israel et al., 1997). Since then, little progress has been made in this field, until the developments of the last few years.

Extensive information on hot subdwarfs can be found in the excellent review by Heber (2009), which, however, does not cover their high-energy properties, having been written before most of the results described below were obtained. The aim of this paper is to review the X-ray properties of hot subdwarfs and discuss their relevance in the context of our understanding of mass-loss from hot stars. After a brief introduction on the X-ray emission from early type stars ${ }^{1}$ and on the current knowledge of mass-loss in hot subdwarfs (Sect. 2), we describe all the available X-ray observations of hot subdwarfs in Sect. 3. The interpretation of these observations, in particular their implications on the stellar wind properties and on the subdwarfs with compact companions, are discussed in Sect. 4 .

\section{Relevance of X-ray observations of hot subdwarfs}

There are two main processes that can lead to the production of X-rays in hot subdwarf stars: wind emission and accretion onto a compact companion. In both cases, X-ray observations can give information on the star properties, although, strictly speaking, the latter process does not involve emission from the hot subdwarf itself. The detection of accretion-powered emission gives the possibility to discover the hot subdwarfs with white dwarf or neutron star companions, which are predicted from evolutionary calculations, but not easily identified with optical observations.

\footnotetext{
${ }^{1}$ An exhaustive coverage of this topic is provided by the other articles of this Special Issue.
} 
It is well known that early-type stars can generate X-rays if their stellar winds contain plasma sufficiently hot to emit in this energy range. In the following, we will call this process "intrinsic" X-ray emission. Extrapolating to lower luminosities the empirical relation between X-ray and bolometric luminosity observed in normal OB stars, $L_{X} / L_{B O L}=10^{-7 \pm 1}$ (Pallavicini et al., 1981; Nazé, 2009), one can estimate the expected X-ray emission for hot subdwarfs. This leads to expected X-ray luminosities of the order of $10^{27-32} \mathrm{erg} \mathrm{s}^{-1}$ and $10^{26-29} \mathrm{erg} \mathrm{s}^{-1}$ for $\mathrm{O}$ and B type subdwarfs, respectively. It must be remembered, however, that the above average relation has a large scatter.

The second possibility is that X-rays are produced by accretion onto a compact companion star, which can be either a white dwarf (WD) or a neutron star (NS) ${ }^{2}$ As a first approximation, the accretion rate can be estimated using the Bondi-Hoyle formalism $3^{3}$ according to which the accretion radius onto an object of mass $M$ is given by $R_{A}=2 G M /\left(V_{W}^{2}+V_{O R B}^{2}\right)$, where $V_{W}$ is the wind velocity and $V_{O R B}$ is the orbital velocity. The mass accretion rate $\dot{M}_{A}$ is related to the relative velocity $V_{R}=\left(V_{W}^{2}+V_{O R B}^{2}\right)^{1 / 2}$ and to the wind density by $\dot{M}_{A}=\pi R_{A}^{2} \rho V_{R}$. The wind density $\rho$ at the position of the compact object can be estimated as $\dot{M}_{\mathrm{W}}=4 \pi a^{2} \rho V_{W}$, where $\dot{M}_{\mathrm{W}}$ is the wind mass-loss rate from the subdwarf and $a$ is the orbital separation. From these relations one obtains the accretion-powered luminosity of a star with mass $M$ and radius $R$

$$
L_{X}=\frac{G M}{R} \dot{M}_{A}=\frac{G M}{R}\left(\frac{R_{A}}{2 a}\right)^{2} \frac{V_{R}}{V_{W}} \dot{M}_{\mathrm{W}} \sim \frac{G M}{R}\left(\frac{R_{A}}{2 a}\right)^{2} \dot{M}_{\mathrm{W}}
$$

Obviously, accretion onto a companion and intrinsic emission are not mutually exclusive, and both processes can occur in binary subdwarfs. In the lack of adequate X-ray data, it can be difficult to distinguish between the two possibilities, but, in both cases, the X-ray emission depends on the properties of the subdwarf's stellar wind. Therefore, X-ray observations provide a new diagnostic tool to investigate the poorly constrained mass-loss processes occurring in these stars.

\footnotetext{
${ }^{2}$ Also binaries composed of a hot subdwarf and a black hole can exist, but their formation is less frequent (Nelemans, 2010).

${ }^{3}$ We assume that the subdwarf radius is smaller than its Roche-lobe; this condition is verified in all the hot subdwarf binaries discussed below.
} 


\subsection{X-ray emission in hot stars}

Stars of $\mathrm{O}$ and B spectral type are sources of soft X-rays with luminosity up to a few $10^{33} \mathrm{erg} \mathrm{s}^{-1}$ and thermal spectra corresponding to plasma temperatures of a few million degrees. As well demonstrated, e.g., by the case of $\zeta$ Puppis (Hervé et al., 2013), high resolution X-ray spectra of the brightest OB stars can provide a wealth of information through the study of emission lines. However, for the majority of the early type stars detected in X-rays, only low resolution spectra are available, which can be adequately fit using simple models of thermal plasma emission. For example, the spectra of a large sample of massive OB stars detected with $X M M-N e w t o n$ were described with either a single Mekal model with $\mathrm{kT} \sim 0.2-1 \mathrm{keV}$, or with the the sum of two or three Mekal models of different temperatures (Nazé, 2009). These data also showed evidence for additiona $4^{4}$ absorption, probably occurring in the stellar wind, in $\mathrm{O}$ stars but not in B stars.

Early-type stars are characterized by winds with typical mass-loss rates $\dot{M}_{\mathrm{W}}$ in the range $10^{-7}-10^{-5} M_{\odot} \mathrm{yr}^{-1}$ and terminal velocities of a few thousands $\mathrm{km} \mathrm{s}^{-1}$. It is believed that the observed X-rays are produced in these stellar winds, where the gas is shock-heated by instabilities (see, e.g., Owocki, 2013, for a review). The main properties of the winds in OB stars are explained in the context of the radiative line-driven wind theory Castor et al., 1975; Kudritzki and Puls, 2000), according to which part of the radial momentum of the photons emitted from the star is transferred to the wind matter through line absorption and reemission. The theory predicts a dependence of $\dot{M}_{\mathrm{W}}$ on the star luminosity approximately given by $\dot{M}_{\mathrm{W}} \propto L^{\alpha}$, with $\alpha \sim 1.5-2$. Since the photon absorption/emission process occurs mainly in the metals present in the wind, the mass-loss rate depends also on the metallicity $Z$, with $\dot{M}_{\mathrm{W}} \propto Z^{b}$ and $b \sim 0.6-0.7$ (Vink et al., 2001). These theoretical scaling laws are generally in good agreement with the observational data. However, some discrepancies have been found in stars with low-density winds, which show mass-loss rates one or two orders of magnitude below the predicted values (Martins et al., 2005; Marcolino et al., 2009). Such discrepancies might be, at least partially, explained by the fact that the UV line diagnostics used in these stars underestimate the actual mass-loss rates. The study of hot subdwarfs in the X-ray band, providing alternative handles on the properties of weak winds, can be of interest in this respect.

\footnotetext{
${ }^{4}$ With respect to the value expected for the interstellar medium along the line of sight.
} 


\subsection{Stellar winds in hot subdwarfs}

As mentioned above, the theory of radiatively-driven winds predicts that, for a given temperature and composition, the mass-loss rate scales with the star luminosity. This leads to the simple expectations that the winds of hot subdwarfs should be weaker than those of main sequence, giant and supergiant OB stars and that sdOs should have stronger winds than sdBs.

These predictions are indeed confirmed by the observations: evidence for stellar winds has been reported for some sdOs, but not yet conclusively for any sdB star. In fact, the mass-loss rates of a few sdOs have been derived from the P-Cygni profiles of the $\mathrm{C}_{I V}$ and $\mathrm{N}_{V}$ lines in the UV, yielding values of $\dot{M}_{\mathrm{W}} \sim 10^{-9}-10^{-8} M_{\odot} \mathrm{yr}^{-1}$ (Hamann et al., 1981; Jeffery and Hamann, 2010; Hamann, 2010). On the other hand, for what concerns sdB stars, the only evidence for mass-loss comes from the observation of a few objects with anomalous profiles of the $\mathrm{H}_{\alpha}$ and HeI lines, interpreted as possible hints of a weak stellar wind (Heber et al., 2003).

Vink and Cassisi (2002) computed models of radiation-driven winds for low mass stars with $10 \mathrm{kK}<T_{\text {eff }}<35 \mathrm{kK}$, obtaining the following relation between the mass-loss rate and the star mass, $M$, luminosity, $L$, and metallicity $Z$ :

$$
\begin{gathered}
\log \dot{M}_{W}=-11.70( \pm 0.08)+1.07( \pm 0.32) \log \left(T_{\text {eff }} / 20 \mathrm{kK}\right) \\
+2.13( \pm 0.09)\left(\log \left(L / L_{\odot}\right)-1.5\right) \\
-1.09( \pm 0.05) \log \left(M / 0.5 M_{\odot}\right)+0.97( \pm 0.04) \log \left(Z / Z_{\odot}\right)
\end{gathered}
$$

The range of validity of this relation $\left(0.5<M / M_{\odot}<0.7,1.3<\log \left(L / L_{\odot}\right)<\right.$ 1.7 , and $\left.0.1<Z / Z_{\odot}<10\right)$ is appropriate for sdB stars. Fig. 1 shows the mass-loss rates as a function of $T_{\text {eff }}$, computed with this relation for the case of $M=0.6 M_{\odot}$ and different values of $L$ and $Z$. Unglaub (2008) computed mass-loss rates for sdBs obtaining values similar to those of Vink and Cassisi $(2002)$ for stars of solar metallicity, but much lower values in the case of $Z<Z_{\odot}$ and high surface gravities.

The wind composition is an important parameter affecting the mass-loss rate, but unfortunately only limited information on the metallicity of hot subdwarfs is available. The above theoretical estimates for $\dot{M}_{W}$ are based on the assumption that the wind behaves as a single fluid, i.e. there is sufficient Coulomb coupling between the metals which are accelerated and the $\mathrm{H}$ and He atoms which constitute the bulk of the wind matter. Such a condition might not be valid in very low-density winds (Krtička and Kubát, 2010). 


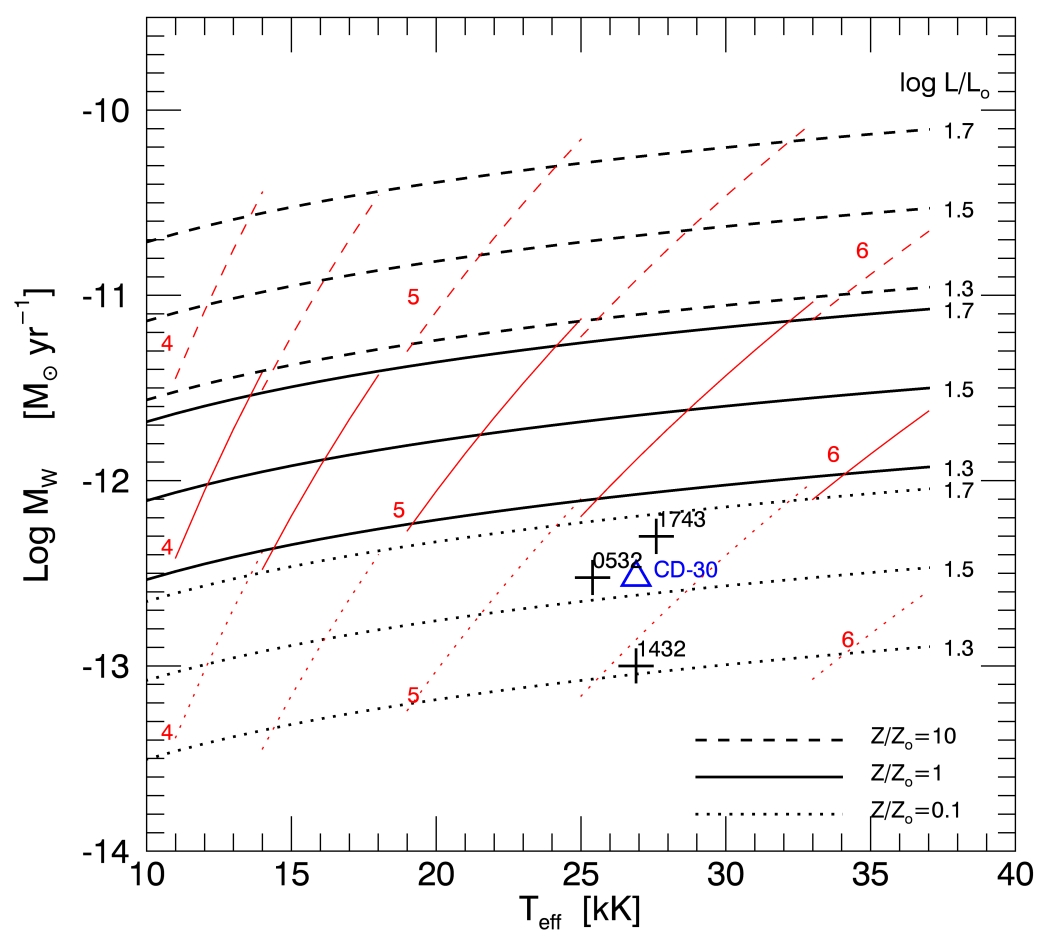

Figure 1: Mass-loss rate as a function of effective temperature, according to the relation of Vink and Cassisi (2002), for a star of $0.6 M_{\odot}$. Each set of lines refers to a different metallicity value $\left(Z=0.1 Z_{\odot}\right.$ dotted lines, $Z=1 Z_{\odot}$ solid lines, $Z=10 Z_{\odot}$ dashed lines $)$ and three luminosity values $\left(\log \left(L / L_{\odot}\right)=1.3,1.5,1.7\right)$. The thin red lines show the corresponding values of $\log g$. The triangle indicates the upper limit, derived from Xray observations, on the mass-loss rate of the sdB star $\mathrm{CD}-30^{\circ} 11223$, which has a WD companion. The crosses indicate the mass-loss rates upper limits derived for three other sdB stars (PG 1432+159, PG 1743+477, HE 0532-4503), with the hypothesis that they have neutron star companions (see Sect.4.3). 


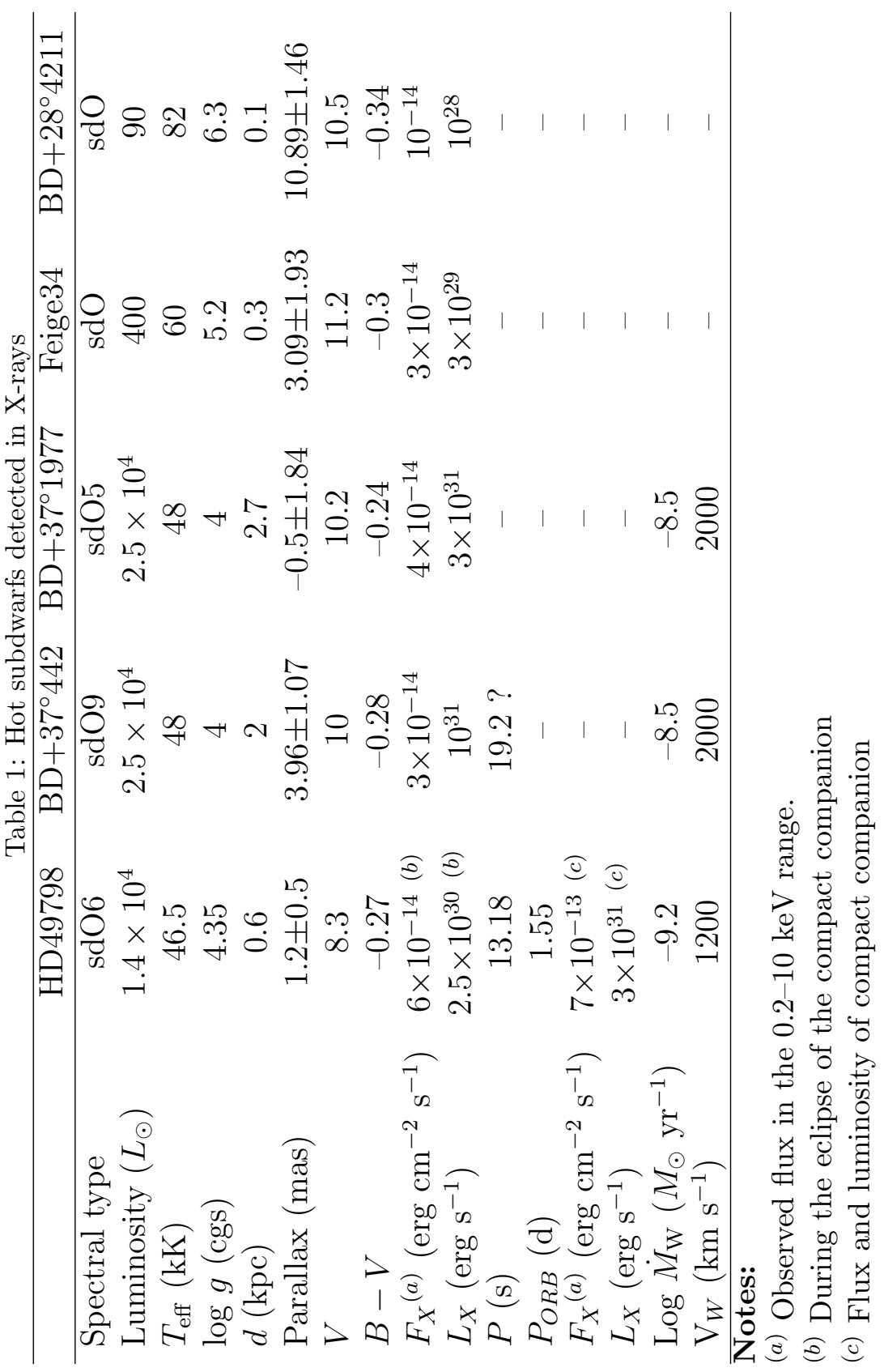




\section{X-ray observations of hot subdwarfs}

The main properties of the five hot subdwarfs that have been detected in the X-ray band are given in Table 1. These stars are individually discussed in the following subsections. In Table 2 we list all the hot subdwarfs that have been observed with sensitive X-ray telescopes (XMM-Newton, Chandra, Swift), but without a significant detection.

\section{1. $H D 49798$}

HD 49798 is the X-ray brightest hot subdwarf and the only one for which most of the observed emission can be unambiguoulsy attributed to accretion onto a compact companion star. Early observations of this bright $(\mathrm{V}=8.3)$, single-lined spectroscopic binary revealed its orbital period of 1.5477 days and led to the measurement of the optical mass function $\mathrm{f}(\mathrm{M})=0.27 \mathrm{M}_{\odot}$ (Thackeray, 1970; Stickland and Lloyd, 1994), but the nature of its unseen companion remained unknown for many years. Extensive optical/UV spectroscopic studies indicated that HD 49798 is a luminous $\left(\sim 5 \times 10^{37} \mathrm{erg} \mathrm{s}^{-1}\right)$ subdwarf of O6 spectral type, with peculiar abundances: it is rich in He and $\mathrm{N}$, while $\mathrm{C}$ is underabundant (see Table 3). These abundances are consistent with evolutionary models according to which HD 49798 is the core of an intially much more massive star which lost its $\mathrm{H}$ envelope, most likely during a common-envelope phase.

X-rays from HD 49798 were first detected in 1979 with the Einstein Observatory, and later shown to be modulated with a period $\mathrm{P}=13.2 \mathrm{~s}$ thanks to a ROSAT observation carried out in 1992 (Israel et al., 1997). Such a short and regular periodicity can only be produced by the rotation of either a WD or a NS. As first suggested by Thackeray (1970), the fact that the companion star is a degenerate object explains why it is not visible in the optical/UV spectra, which are dominated by the emission of the much larger and brighter sdO star.

HD 49798 has been repeatedly observed with the XMM-Newton and Swift satellites. These data, spanning the years from 2002 to 2014, as well as the few previous observations with other satellites, indicate a remarkably stable X-ray emission. No significant variations were seen in the source flux, spectral shape, pulse profile, and spin period, with a limit on the period derivative of $|\dot{P}|<6 \times 10^{-15} \mathrm{~s} \mathrm{~s}^{-1}$ (Mereghetti et al., 2013).

A dynamical measurement of the masses of HD 49798 and its compact companion has been obtained by the analysis of the orbitally-induced phase 

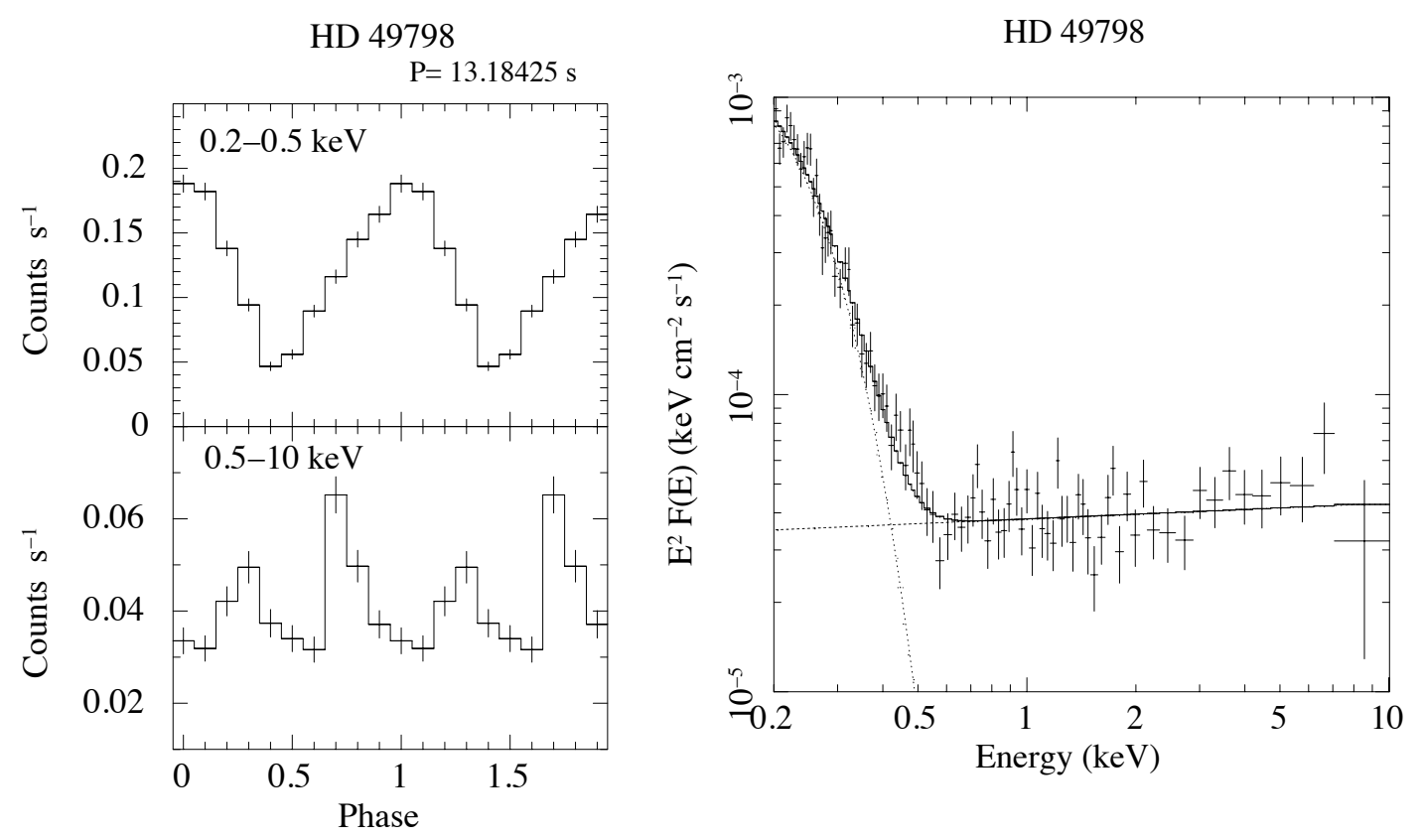

Figure 2: Results for HD 49798 obtained with the EPIC instrument on XMM-Newton. Left panel: X-ray light curve in two energy ranges folded at the spin period of $13.2 \mathrm{~s}$. Right panel: X-ray spectrum fitted with a blackbody plus power law model (observation performed in October 2014).

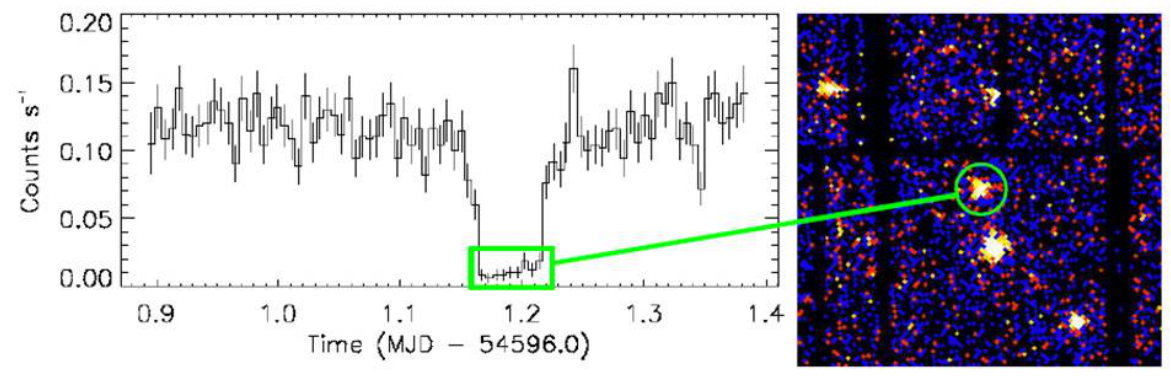

Figure 3: Left panel: X-ray light curve of HD 49798 obtained in May 2008 showing the eclipse with duration of 1.3 hours. Right panel: X-ray image $(0.2-10 \mathrm{keV})$ accumulated during the eclipse in which emission from HD 49798 is clearly visible. 
delays of the X-ray pulses, combined with the optical mass function and with the system inclination estimated from the duration of the X-ray eclipse (Mereghetti et al., 2009). The measured mass of HD 49798 is $1.50 \pm 0.05$ $M_{\odot}$, among the highest seen in hot subdwarfs, while that of its compact companion is $1.28 \pm 0.05 M_{\odot}$, consistent with either a NS or a massive WD.

Both the timing and spectral properties indicate that the X-ray emission from the compact companion of HD 49798 consists of two different components (Mereghetti et al., 2011b, 2013). The spectrum (Fig. 2, right panel) comprises a soft thermal component, well fit by a blackbody of temperature $\mathrm{kT}_{B B} \sim 30 \mathrm{eV}$ and a hard component which dominates the emission above $0.5 \mathrm{keV}$. The latter is well fit by either a power law of photon index $\Gamma \sim 2$ or a thermal bremsstrahlung with temperature $\mathrm{kT}_{B R} \sim 4 \mathrm{keV}$. The folded pulse profile is single-peaked and strongly modulated below $0.5 \mathrm{keV}$, where the soft thermal component dominates, while it is double-peaked at higher energies (see Fig. 2, left panel). The striking difference in the pulse profile at low and hard energies suggests an interpretation in terms of two separate physical components, e.g. thermal emission from a hot spot on the star surface and non-thermal emission with a more complex beam pattern produced in the magnetosphere. The luminosity of $\mathrm{L}_{X}=3 \times 10^{31} \mathrm{erg} \mathrm{s}^{-1}(0.2-10 \mathrm{keV}$, $\mathrm{d}=0.6 \mathrm{kpc}$ ) indicates that the accreting companion of HD 49798 is most likely a $\mathrm{WD}^{5}$, since accretion onto a NS would result in a luminosity larger by a factor $\mathrm{R}_{W D} / \mathrm{R}_{N S} \sim 300$ (see Eq.(1)).

As shown in Fig. 3, X-rays from the HD 49798 binary system are detected also when the compact object is eclipsed by the sdO star, which has a radius $\left(1.45 \pm 0.25 R_{\odot}\right.$, Kudritzki and Simon (1978) ) much larger than that of its companion $(\sim 3000 \mathrm{~km}$ for a massive WD or $\sim 10 \mathrm{~km}$ for a NS). The X-ray emission during the eclipse is fainter, by a factor $\sim 10$, and harder than that seen in the orbital phases outside the eclipse. Its spectral properties and likely origin as intrinsic emission from HD 49798 are discussed in Sect. 4.1.

\section{2. $B D+34442$}

$\mathrm{BD}+37^{\circ} 442$ is a luminous $\left(\sim 10^{38} \mathrm{erg} \mathrm{s}^{-1}\right)$, extremely helium-rich sdO star (Rebeirot, 1966; Husfeld, 1987). Its temperature, luminosity, and surface gravity, as well as its mass-loss properties (Jeffery and Hamann, 2010), are

\footnotetext{
${ }^{5}$ This is also supported by the emission radius derived from the blackbody fit, $\sim 40 \mathrm{~km}$, larger than that of a NS and consistent with a hot spot on the surface of a WD.
} 
similar to those of HD 49798 (see Table 1). However, contrary to HD 49798, no evidence for it being member of a binary system has been reported in the literature. A recent spectroscopic search for a binary signature did not detect any radial velocity variation on timescales from hours to months, down to a level of a few $\mathrm{km} \mathrm{s}^{-1}$ (Heber et al., 2014).

Motivated by the X-ray detection of HD 49798 during eclipse, we searched for intrinsic X-ray emission from this allegedly single sdO star with an $X M M$ Newton observation carried out in August 2011. Soft X-ray emission from $\mathrm{BD}+37^{\circ} 442$ was clearly detected, with a flux of $3 \times 10^{-14} \mathrm{erg} \mathrm{cm}^{-2} \mathrm{~s}^{-1}(0.2-1$ $\mathrm{keV}$ ). Quite unexpectedly, a timing analysis of the X-ray data revealed (at $3 \sigma$ statistical significance) a periodicitiy at $19.2 \mathrm{~s}$ (La Palombara et al., 2012). Fitting the X-ray spectrum of $\mathrm{BD}+37^{\circ} 442$ with the sum of a soft blackbody and a power law component yields parameters similar to those of HD 49798, but with larger uncertainties: fixing the photon index to $\Gamma=1$ or 3 , blackbody temperatures in the range $\sim 30-68 \mathrm{eV}$ are obtained. The poorly constrained blackbody temperature and normalization do not permit a precise estimate of the total X-ray luminosity, which could be in the range from $\sim 10^{32}$ to $10^{35} \mathrm{erg} \mathrm{s}^{-1}$. If confirmed, the periodicity at $19.2 \mathrm{~s}$ detected in $\mathrm{BD}+37^{\circ} 442$ requires the presence of a compact companion, which, considering the large uncertainty on the X-ray luminosity, could be either a WD or a NS.

If $\mathrm{BD}+37^{\circ} 442$ is indeed in a binary system, the lack of radial velocity variations in its spectrum is puzzling. Three explanations are possible: a) a very small orbital inclination; b) a very long orbital period (> several months); c) the reported pulsations are not real. Case a) seems unlikely because the large projected rotational velocity $\left(60 \mathrm{~km} \mathrm{~s}^{-1}\right.$, Heber et al. (2014)) would imply a significant misalignment between the orbital and the star rotation axis. Also case b) presents some difficulties because a large orbital separation would probably result in a low mass accretion rate, unless the orbit is highly eccentric, causing X-ray emission mainly close to periastron passage (in this case time variability of the $\mathrm{X}$-ray emission is expected). More observations are needed to confirm the presence of pulsations and investigate more deeply the different possibilities. As discussed in Sect. 4.1, the X-ray spectrum of $\mathrm{BD}+37^{\circ} 442$ can also be fit by a sum of thermal plasma models (see Table 3 and Fig. 4, middle panel), consistent with intrinsic emission from an isolated sdO. 
Table 2: Upper limits on the X-ray emission from hot subdwarfs

\begin{tabular}{|c|c|c|c|c|c|}
\hline Name & Type & $\begin{array}{c}d \\
(\mathrm{kpc})\end{array}$ & $\begin{array}{c}P_{\mathrm{ORB}} \\
(\text { days })\end{array}$ & $\begin{array}{c}L_{X}(a) \\
\left(\operatorname{erg~s}^{-1}\right)\end{array}$ & $\operatorname{Ref}^{(b)}$ \\
\hline $\mathrm{CD}-30^{\circ} 11223$ & $\mathrm{sdB}+\mathrm{WD}$ & 0.36 & 0.049 & $<1.5 \times 10^{29}$ & 2 \\
\hline PG $1043+760$ & $\mathrm{sdB}+\mathrm{WD} ?$ & 0.12 & 0.66 & $<6.8 \times 10^{30}$ & 1 \\
\hline PG $1432+159$ & $\mathrm{sdB}+\mathrm{NS} / \mathrm{BH} ?$ & 0.22 & 0.8 & $<9.9 \times 10^{30}$ & 1 \\
\hline PG $2345+318$ & sdB+WD? & 0.24 & 0.9 & $<1.3 \times 10^{31}$ & 1 \\
\hline HE 0532-4503 & $\mathrm{sdB}+\mathrm{NS} / \mathrm{BH} ?$ & 0.27 & 2.8 & $<7.4 \times 10^{31}$ & 1 \\
\hline CPD -64 481 & $\mathrm{sdB}+\mathrm{WD} ?$ & 0.28 & 0.21 & $<6.8 \times 10^{29}$ & 1 \\
\hline PG $1101+249$ & $\mathrm{sdB}+\mathrm{WD} / \mathrm{NS} / \mathrm{BH} ?$ & 0.35 & 0.39 & $<1.7 \times 10^{30}$ & 1 \\
\hline PG 1232-136 & $\mathrm{sdB}+\mathrm{BH} ?$ & 0.363 & 0.57 & $<5.0 \times 10^{29}$ & 2 \\
\hline GD 687 & $\mathrm{sdB}+\mathrm{WD} ?$ & 0.38 & 1.1 & $<1.0 \times 10^{31}$ & 1 \\
\hline HE 0929-0424 & $\mathrm{sdB}+\mathrm{WD} / \mathrm{NS} / \mathrm{BH} ?$ & 0.44 & 1.9 & $<2.9 \times 10^{31}$ & 1 \\
\hline PG $1743+477$ & $\mathrm{sdB}+\mathrm{NS} / \mathrm{BH} ?$ & 0.52 & 1 & $<1.8 \times 10^{31}$ & 1 \\
\hline PG $0101+039$ & sdB+WD? & 0.57 & 0.33 & $<1.0 \times 10^{30}$ & 1 \\
\hline TON S 183 & $\mathrm{sdB}+\mathrm{WD} ?$ & 0.83 & 0.54 & $<2.5 \times 10^{30}$ & 1 \\
\hline $\mathrm{BD}+75^{\circ} 325$ & $\mathrm{sdO}$ & 0.136 & - & $<5.8 \times 10^{28}$ & 3 \\
\hline $\mathrm{BD}+25^{\circ} 4655$ & $\mathrm{sdO}$ & 0.11 & - & $<5.1 \times 10^{28}$ & 3 \\
\hline $\mathrm{BD}-22^{\circ} 3804$ & $\mathrm{sdO}$ & 0.185 & - & $<9.7 \times 10^{28}$ & 3 \\
\hline $\mathrm{BD}+39^{\circ} 3226$ & $\mathrm{sdO}$ & 0.235 & - & $<1.5 \times 10^{29}$ & 3 \\
\hline $\mathrm{BD}-03^{\circ} 2179$ & $\mathrm{sdO}$ & 0.631 & - & $<2.4 \times 10^{30}$ & 3 \\
\hline $\mathrm{CD}-31^{\circ} 4800$ & $\mathrm{sdO}$ & 0.132 & - & $<5.1 \times 10^{28}$ & 3 \\
\hline $\mathrm{BD}+48^{\circ} 1777$ & $\mathrm{sdO}$ & 0.163 & - & $<1.2 \times 10^{29}$ & 3 \\
\hline $\mathrm{LS} \mathrm{V}+22^{\circ} 38$ & $\mathrm{sdO}$ & 0.18 & - & $<1.5 \times 10^{29}$ & 3 \\
\hline LS IV $-12^{\circ} 1$ & $\mathrm{sdO}$ & 0.4 & - & $<4.4 \times 10^{29}$ & 3 \\
\hline LSE 153 & $\mathrm{sdO}$ & 0.25 & - & $<3.6 \times 10^{29}$ & 3 \\
\hline LSS 1275 & $\mathrm{sdO}$ & $<1$ & - & $<2.1 \times 10^{31}$ & 3 \\
\hline LSE 263 & $\mathrm{sdO}$ & 0.25 & - & $<4.6 \times 10^{29}$ & 3 \\
\hline $\mathrm{BD}+18^{\circ} 2647$ & $\mathrm{sdO}$ & 0.275 & - & $<3.3 \times 10^{29}$ & 3 \\
\hline LSE 21 & $\mathrm{sdO}$ & 0.05 & - & $<7.1 \times 10^{27}$ & 3 \\
\hline $\mathrm{LS} \mathrm{IV}+10^{\circ} 9$ & $\mathrm{sdO}$ & 0.23 & - & $<1.5 \times 10^{29}$ & 3 \\
\hline LS I +63ํ 198 & $\mathrm{sdO}$ & 0.2 & - & $<1.4 \times 10^{29}$ & 3 \\
\hline
\end{tabular}

Notes:

(a) Luminosity in the $0.2-10 \mathrm{keV}$ range, assuming a thermal spectrum similar to that of $\mathrm{BD}+37^{\circ} 442$.

(b) (1) Mereghetti et al. (2011a); (2) Mereghetti et al. (2014); (3) La Palombara et al. (2014); 
HD 49798 (eclipse)

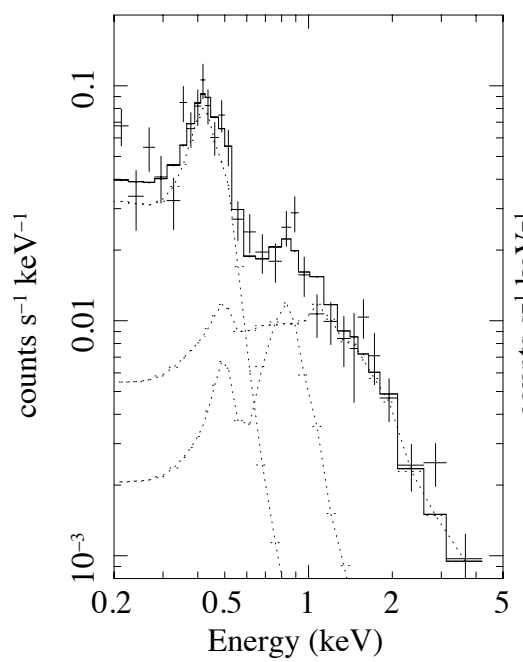

$\mathrm{BD}+37442$

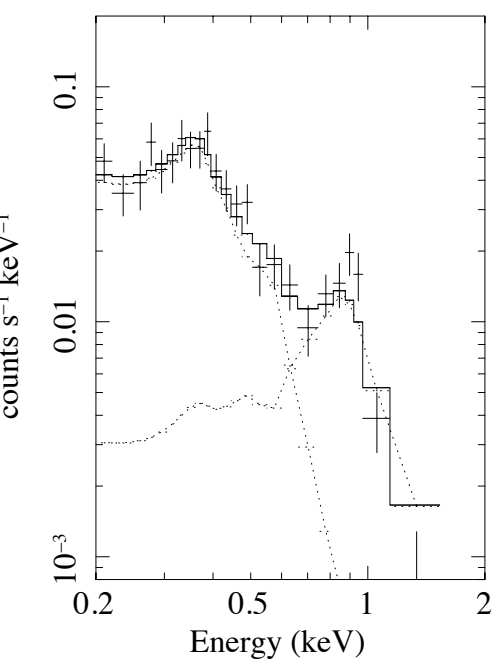

BD +37 1977

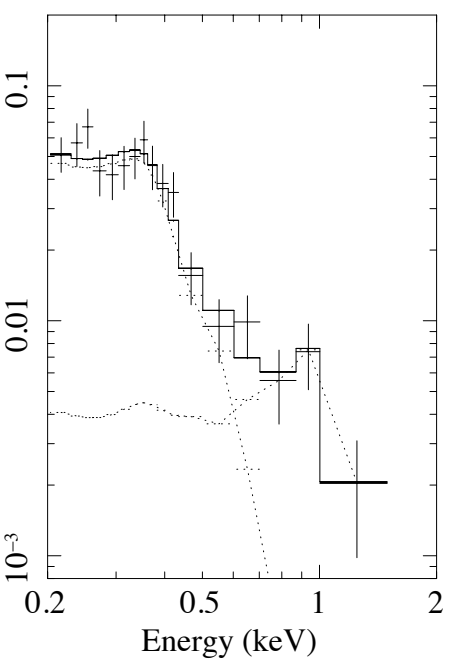

Figure 4: Left panel: XMM-Newton EPIC (MOS+pn) spectrum of HD 49798 during the eclipse. The solid line shows the best fit with three thermal plasma models (Mekal) with temperatures $\mathrm{kT}_{1}=0.11 \mathrm{keV}, \mathrm{kT}_{2}=0.57 \mathrm{keV}$, and $\mathrm{kT}_{3}=4 \mathrm{keV}$ (fixed). The spectrum has been obtained by summing observations performed in 2008, 2011 and 2013, giving a total exposure time of $25 \mathrm{ks}$. Middle panel: XMM-Newton EPIC (MOS+pn) spectrum of $\mathrm{BD}+37^{\circ} 442$ fitted with two Mekal models with temperatures $\mathrm{kT}_{1}=0.11 \mathrm{keV}$ and $\mathrm{kT}_{2}=0.65$ $\mathrm{keV}$. Right panel: XMM-Newton pn spectrum of $\mathrm{BD}+37^{\circ} 1977$ fitted with two Mekal models with temperatures $\mathrm{kT}_{1}=0.13 \mathrm{keV}$ and $\mathrm{kT}_{2}=0.79 \mathrm{keV}$. In all the spectra the interstellar absorption and the abundances have been fixed at the values given in Table 3 . The dashed lines show the individual Mekal components. 
Table 3: Spectral parameters of three sdO stars fitted with the sum of two or three thermal spectra.

\begin{tabular}{lccc}
\hline & HD 49798 & $\mathrm{BD}+37^{\circ} 442$ & $\mathrm{BD}+37^{\circ} 1977$ \\
\hline $\mathrm{N}_{H}\left(\mathrm{~cm}^{-2}\right)$ & $5 \times 10^{20}$ & $5 \times 10^{20}$ & $10^{20}$ \\
$\mathrm{kT}_{1}(\mathrm{keV})$ & 0.11 & 0.11 & 0.13 \\
$\mathrm{kT}_{2}(\mathrm{keV})$ & 0.57 & 0.65 & 0.79 \\
$\mathrm{kT}_{3}(\mathrm{keV})$ & 4. & - & - \\
\hline
\end{tabular}

\begin{tabular}{lccc} 
Abundances $^{(a)}$ & & & \\
$X_{\mathrm{H}}$ & 0.19 & 0.0013 & 0.0013 \\
$X_{\mathrm{He}}$ & 0.78 & 0.96 & 0.96 \\
$X_{\mathrm{C}}$ & 0.0001 & 0.025 & 0.025 \\
$X_{\mathrm{N}}$ & 0.025 & 0.003 & 0.003 \\
$X_{\mathrm{O}}$ & 0.0028 & 0.005 & 0.005 \\
$X_{\mathrm{Si}}$ & 0.001 & 0.0008 & 0.0008 \\
$X_{\mathrm{Fe}}$ & 0.0011 & 0.0006 & 0.0006 \\
\hline$(a)$ mass fraction. & & &
\end{tabular}

\section{3. $B D+3701977$}

This luminous and He-rich sdO has been dubbed the spectroscopic twin of $\mathrm{BD}+37^{\circ} 442$, because the optical/UV spectral properties of these two stars are very similar. The X-ray emission from $\mathrm{BD}+37^{\circ} 1977$ was discovered thanks to a Chandra survey of a flux-limited sample of sdOs (La Palombara et al., 2014) and recently studied in more detail with a dedicated $X M M$ Newton observation in which it was detected in the $0.15-1.5 \mathrm{keV}$ range (La Palombara et al., 2015). Its very soft X-ray spectrum is well fit by the sum of two thermal plasma models with temperatures of about $0.13 \mathrm{keV}$ and 0.8 $\mathrm{keV}$, provided that adequate elemental abundances are used (see details in Table 3). The X-ray luminosity is $3.3 \times 10^{31} \mathrm{erg} \mathrm{s}^{-1}$ (for $\mathrm{d}=2.7 \mathrm{kpc}$ ). A search for periodicities gave negative results, but, given the faint X-ray flux, the upper limits on the pulsed fraction are not particularly constraining. $\mathrm{BD}+37^{\circ} 1977$ is a bright and relatively well studied star in the optical/UV bands and no radial velocity variations have been reported in the literature. It is thus natural to interpret these results in terms of intrinsic X-ray emission from the sdO star. 


\section{4. $B D+28^{\circ} 4211$ and Feige 34}

$\mathrm{BD}+28^{\circ} 4211$ and Feige 34 are the only other sdOs detected in the Chandra survey carried out by La Palombara et al. (2014). Being very faint sources (X-ray fluxes of a few $10^{-14} \mathrm{erg} \mathrm{s}^{-1}$ ), they yielded only a few counts in these short observations: enough for a significant detection in the low-background Chandra HRC-I instrument, but insufficient for a timing analysis.

The parallax of $\mathrm{BD}+28^{\circ} 4211$ obtained with Hipparcos implies a very small distance. Therefore, this star, with an X-ray luminosity of only $\sim 10^{28} \mathrm{erg}$ $\mathrm{s}^{-1}$, is the closest hot subdwarf detected in the X-ray band. Note that the angular resolution of the Chandra data is sufficiently good to unambiguosly associate the observed X-ray emission to $\mathrm{BD}+28^{\circ} 4211$ and not to a faint companion star lying at an angular distance of only 2.8 arcsec (Massey and Gronwall, 1990). A detailed analysis of the UV and optical spectra of $\mathrm{BD}+28^{\circ} 4211$ with NLTE models indicates a surface gravity in the range $6.1<\log g<6.5$ (Latour et al., 2013, 2015). Thus, contrary to the three objects discussed in the previous subsections, this star belongs to the class of low-luminosity, compact sdOs.

The same might be true for Feige 34, for which Thejll et al. (1995) derived $\log g=6.8$ (this is, however, at variance with the value $\log g=5.2$ reported by Werner et al. (1998)). Feige 34 has an infrared excess which might result either from free-free emission in a wind or from a late type companion star. The latter possibility is favored by the analysis of Thejll et al. (1995), who estimate that the putative companion should be of M2 spectral type. Since also late-type stars are known X-ray emitters, it cannot be excluded that (part of) the observed X-ray emission is due to the putative companion.

\section{5. sdBs with (candidate) compact companions}

Up to now, no sdB star has been detected at X-ray energies. The recent observations carried out with sensitive satellites focussed on sdBs in binaries with either confirmed or candidate compact companions. Although none of the observed targets were detected, these observations are relevant because they provide upper limits that are orders of magnitude smaller than those previously available from the ROSAT All Sky Survey.

CD $-30^{\circ} 11223$ is the most interesting sdB for which a deep upper limit on the X-ray luminosity has been obtained. Contrary to the case of the other sdB binaries discussed below, the presence of a compact companion is certain: it is a WD with a dynamically-measured mass of $0.7 M_{\odot}$, orbiting the subdwarf star with a period of only $1.2 \mathrm{hr}$, the shortest known among sdB 
binaries (Vennes et al., 2012, Geier et al., 2013). A 50 ks long observation with $X M M$-Newton yielded a luminosity upper limit of $\mathrm{L}_{X} \sim 1.5 \times 10^{29} \mathrm{erg}$ $\mathrm{s}^{-1}$ (for $\mathrm{d}=364 \mathrm{pc}$ ), which, as discussed in Sect. 4.3, provides interesting constraints on the mass-loss rate from this sdB (Mereghetti et al., 2014).

The presence of a compact companion has been suggested for several other sdB stars. These systems with "candidate" compact companions have been selected by means of radial and rotational velocity measurements of singlelined spectroscopic binaries (Geier et al., 2010). The assumption of synchronous rotation in these systems allows one to estimate the orbit inclination and thus to set a lower limit to the mass of the companion. If this limit exceeds the value expected for a late-type main-sequence star, it is likely that the companion is a WD or a NS. A search for X-ray emission from a sample of twelve such systems, chosen among those with the lowest interstellar absorption and shortest orbital periods, was carried out with the Swift satellite (Mereghetti et al. 2011a). Upper limits in the range $\sim 10^{30}-10^{31} \mathrm{erg} \mathrm{s}^{-1}$ were obtained for their X-ray luminosities (Table 2). The implications for the wind mass-loss rates of these sdBs are discussed in Sect. 4.3.

\section{Discussion}

\subsection{Intrinsic $X$-ray emission from hot subdwarfs}

In Fig. 5 we compare the X-ray and bolometric luminosities of the sdO stars to those of the normal O-type stars seen in the ROSAT All Sky Survey (Berghoefer et al., 1996). For the case of HD 49798 we consider here only the X-rays observed during the eclipse of its compact companion, which are most likely due to intrinsic emission from the sdO star ${ }^{6}$. It is clear that the luminosities of the five sdOs detected in X-rays, as well as the upper limits of the undetected ones, are consistent with an extrapolation of the average relation $L_{X} / L_{B O L}=10^{-7 \pm 1}$ followed by the more luminous O-type stars (Pallavicini et al., 1981; Nazé, 2009).

From the X-ray spectral point of view, the sdOs are similar to the normal O-type stars. As shown in Fig. 4, good fits to the X-ray spectra of HD 49798 (during the eclipse), $\mathrm{BD}+37^{\circ} 442$ and $\mathrm{BD}+37^{\circ} 1977$ can be obtained with

\footnotetext{
${ }^{6}$ Actually it cannot be completely excluded that (some of) the X-rays detected during eclipse are due to emission from the compact object reprocessed in the wind of the hot subdwarf (see discussion in Mereghetti et al. (2013)); this might explain its harder spectrum compared to the other sdO stars.
} 


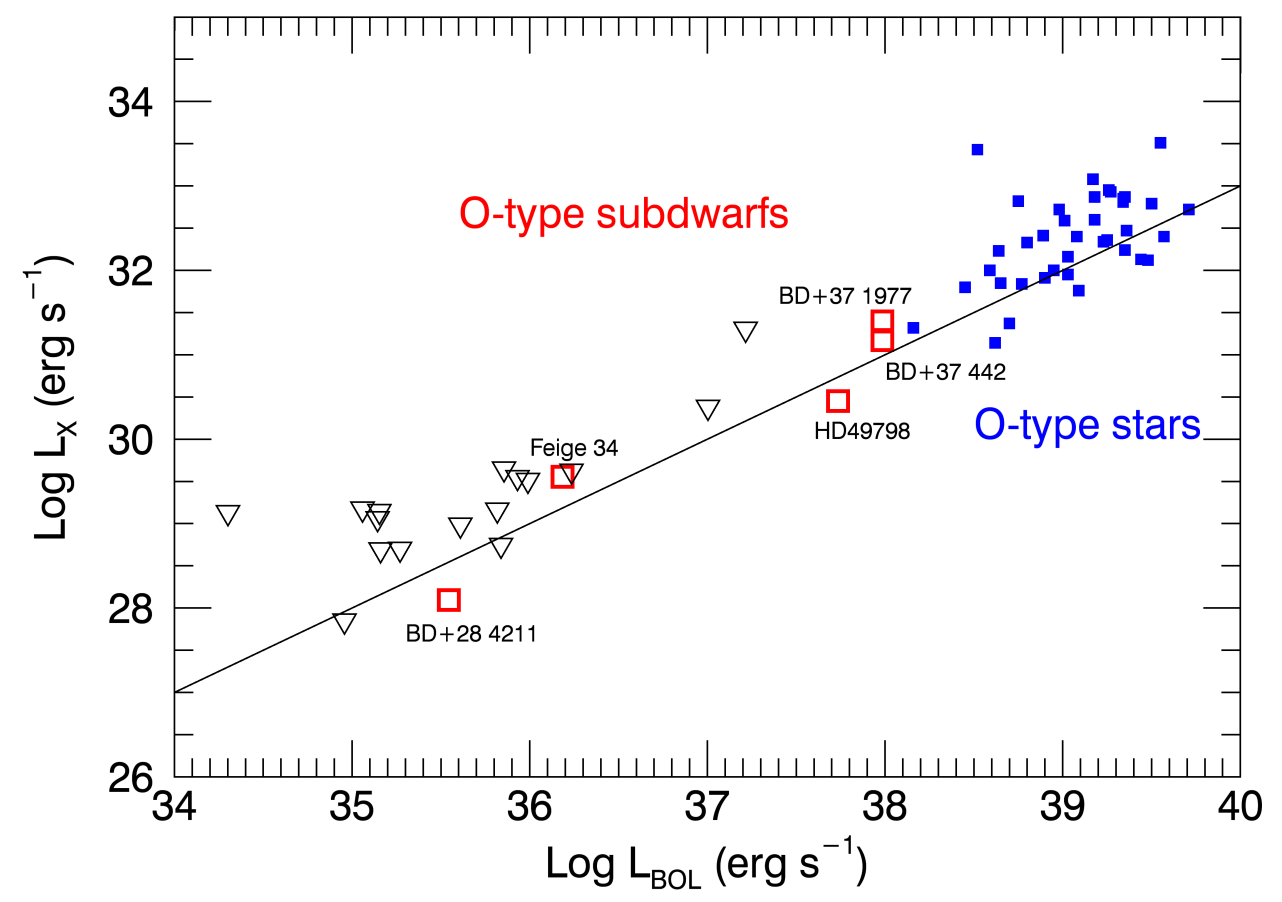

Figure 5: X-ray versus bolometric luminosity for hot subdwarfs. The five red squares indicate the sdO detected in X-rays, while the upper limits for the non-detected sdOs are shown by the black triangles. The blue squares show for comparison the $\mathrm{O}$ stars detected in the ROSAT All Sky Survey (Berghoefer et al., 1996). The line indicates the relation $L_{X} / L_{B O L}=10^{-7}$. 
the sum of two or three plasma emission models with different temperatures, provided that the proper element abundances are adopted. It is interesting to note that these three X-ray emitting sdOs are among the few hot subdwarfs for which evidence of mass-loss has been reported. No spectral information is available for the two remaining faint sdOs, which were only observed with the Chandra HRC instrument.

These findings suggest that the process of X-ray emission due to wind instabilities and shock heating is not restricted to the dense winds of the luminous and massive hot stars, but it can also operate in the weak winds of sdO stars. The five sdOs detected in X-rays have rather different atmospheric compositions: $\mathrm{BD}+37^{\circ} 442$ and $\mathrm{BD}+37^{\circ} 1977$ have almost pure $\mathrm{He}$ atmospheres and are C-rich (Bauer and Husfeld, 1995; Jeffery and Hamann, 2010); HD 49798 has one atom of He for each of $\mathrm{H}$ and is N-rich (Kudritzki and Simon, 1978); the compact sdO BD $+28^{\circ} 4211$ has solar He and subsolar to solar abundances of several metals (Latour et al., 2013, 2015); finally, He is slightly deficient for Feige 34, while $\mathrm{Fe}$ and $\mathrm{Ni}$ are enhanced by factors of 10 and 70 (Werner et al., 1998). These characteristics can in principle cause differences in their winds which might be explored with future, more sensitive X-ray observations.

\subsection{X-ray emission from compact companions: the case of HD 49798}

While the possible presence of a compact companion in $\mathrm{BD}+37^{\circ} 442$ needs a confirmation of the reported $19 \mathrm{~s}$ periodicity, there is no doubt that the spectroscopic binary HD 49798 hosts either a WD or a NS spinning with a period of $13.2 \mathrm{~s}$. As discussed below, accretion onto this compact object is the most natural explanation for the observed pulsed X-rays. The presence of a power-law spectral component rules out a purely thermal emission powered by the internal heat of a cooling NS, while the possibility of rotationpowered emission seems unlikely. The X-ray luminosity of a rotation-powered NS or WD with spin period $\mathrm{P}=2 \pi / \Omega$ is $L_{X}=\eta_{X} \mathrm{I} \Omega \dot{\Omega}$, where $\eta_{X}$ is the efficiency of conversion of rotational energy into radiation and $I$ is the moment of inertia. If the HD 49798 companion is a NS $\left(I_{\mathrm{NS}} \sim 10^{45} \mathrm{~g} \mathrm{~cm}^{2}\right)$, the minimum spin-down rate required to power the observed luminosity is $\dot{P}_{\text {min }}=6 \times 10^{-15}\left(L_{X} / 10^{32} \mathrm{erg} / \mathrm{s}\right)(0.001 / \eta) \mathrm{s} \mathrm{s}^{-1}$, barely compatible with the current upper limit $|\dot{P}|<6 \times 10^{-15} \mathrm{~s} \mathrm{~s}^{-1}$ (Mereghetti et al., 2013). Thus rotation-powered emission from a NS can be ruled out, unless an implausibly high efficiency is invoked. In the case of a WD companion $\left(I_{\mathrm{WD}} \sim 10^{50}\right.$ 
g $\mathrm{cm}^{2}$ ), the required $\dot{P}_{\text {min }}$, being a factor $\sim 10^{5}$ smaller, would be compatible with the observations. A strongly magnetized WD could accelerate relativistic particles, likewise a radio pulsar, thus producing non-thermal magnetospheric X-ray emission. Although the existence of rotation-powered "WD-pulsars" has been proposed (e.g. Usov, 1988), none have been found so far. Also considering that the energetically-dominant thermal component in the HD 49798 X-ray spectrum would be difficult to explain in this scenario, we conclude that the possibility of rotation-powered emission is quite unlikely and concentrate in the following on the accretion scenario.

Independent on the WD or NS nature of the companion star, HD 49798 does not fill its Roche-lobe. Therefore, accretion in this binary occurs by capture of the sdO stellar wind and the expected X-ray luminosity can be estimated with Eq. (1). Mereghetti et al. (2009), based on the wind parameters of HD 49798 available at that time (Hamann et al., 1981), concluded that the compact object is a WD, since an accreting NS would have been more luminous than observed. An updated estimate of the mass-loss rate ( $\log \dot{M}_{\mathrm{W}}=$ $\left.-9.2 \mathrm{yr}^{-1}\right)$ and wind terminal velocity $\left(V_{W}=1200 \mathrm{~km} \mathrm{~s}^{-1}\right)$ has been recently obtained (W. Hamann, private communication, for $\mathrm{d}=0.6 \mathrm{kpc}$ ). With these values and a radius of $3000 \mathrm{~km}$, appropriate for a massive WD, eq. (1) gives a luminosity of $L_{X} \sim 10^{31} \mathrm{erg} \mathrm{s}^{-1}$, which is in reasonable agreement with the observed value of $L_{0.2-10 \mathrm{keV}}=3 \times 10^{31}\left(\frac{d}{0.6 \mathrm{kpc}}\right)^{2} \mathrm{erg} \mathrm{s}^{-1}$. For the alternative possibility of an accreting NS $(\mathrm{R}=10 \mathrm{~km})$, eq. (1) would give $L_{X} \sim 3 \times 10^{33}$ $\operatorname{erg~} \mathrm{s}^{-1}$. Even taking into account that the total luminosity, including the unobserved contribution of the blackbody component below $0.2 \mathrm{keV}$, could be a factor $\sim 4$ higher than the above value, it seems difficult to reconcile the expected NS luminosity with the observations, unless the source distance is greatly underestimated.

Iben and Tutukov (1994) discussed an evolutionary path leading to a system like HD 49798. This involves an initial binary with masses of $\sim 8-9 M_{\odot}$ in which the WD, formed from the originally more massive star, is engulfed in the envelope of the secondary, when the latter expanded to become a red-giant with a non degenerate He core. This leads to the expulsion of the common envelope and to the emergence of a much more compact binary, composed of a CO or ONe WD and a He-star, as currently observed. HD 49798 is now within its Roche-lobe, but it will expand again in the future, transferring He-rich matter onto its companion at a higher rate. If the companion is indeed a massive WD, this could lead to a type Ia supernova explosion or to 
the formation of a rapidly spinning NS through accretion-induced collapse (Wang and Han, 2012; Hurley et al., 2010; Tauris et al., 2013). Being the descendent of intermediate mass stars $\left(\sim 8-9 M_{\odot}\right)$, the HD 49798 binary would be the progenitor of a type Ia supernova with a short delay time, unless, as suggested by Di Stefano et al. (2011), the explosion is delayed due to the fast rotation of the WD. In the alternative case of accretion-induced collapse, the resulting NS would probably have a high rotational velocity. This would be an evolutionary path to create millisecond pulsars without going through recycling in a low mass X-ray binary (Bhattacharya and van den Heuvel, 1991). Recently, Liu et al. (2015) performed population synthesis computations of different evolutionary channels leading to the formation of ONe WD + He-star binaries. They found that systems with long orbital period and high He-star mass, as observed in HD 49798, are rarely formed and conclude that the companion of HD 49798 is more likely to be a CO WD, which can reach Chandrasekhar mass limit and produce a type Ia SN after a few $10^{4}$ years of mass transfer, as computed by Wang and Han (2010).

\subsection{Limits on the mass-loss rate from hot subdwarfs}

The non-detection of accretion-powered X-ray emission in binaries containing compact objects (Sect. 3.5) can be used to constrain the properties of stellar winds from the hot subdwarfs in these systems. Thanks to their higher X-ray efficiency, binaries containing NSs can provide more stringent limits than those containing WDs. Although no confirmed NS+sd system has been found yet, a few candidates which deserve further study have been proposed (Geier et al. 2010). We note that the constraints on the stellar mass-loss derived in this way are affected by a few uncertainties, such as the source distances, the X-ray spectrum assumed to convert count rates to fluxes, the accretion model and geometry used to relate the X-ray luminosity to the mass accretion rate. With these caveats in mind, we can discuss the results obtained for the WD+sdB binary CD -30¹1223 (Mereghetti et al. 2014) and for a dozen of other sdB binaries which are suspected to host compact objects (Mereghetti et al. 2011a).

Under the assumption of Bondi-Hoyle accretion (eq. (1)), and assuming a power law spectrum with photon index $\Gamma=2$, a $3 \sigma$ upper limit of $\dot{M}_{\mathrm{W}} \sim$ $3 \times 10^{-13} M_{\odot} \mathrm{yr}^{-1}$ was derived for the mass-loss rate of $\mathrm{CD}-30^{\circ} 11223$. The distance of this source is well known (364 pc, Geier et al. (2013)), thus the main uncertainty is related to the assumed X-ray spectrum. As discussed

in Mereghetti et al. (2014), any thermal spectrum with temperature in the 

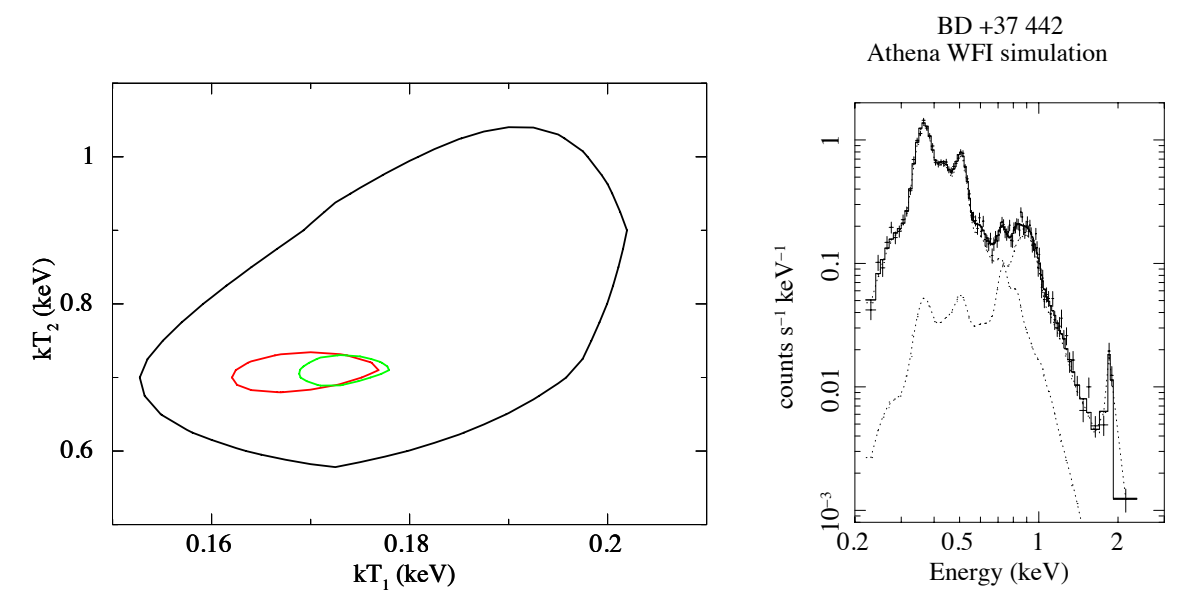

Figure 6: Left panel: Contour plots showing the uncertainty on the temperatures of the two thermal components in the X-ray spectrum of $\mathrm{BD}+37^{\circ} 442$. The black line indicates the current error region ( $2 \sigma$ confidence level) obtained with $X M M$-Newton data. The red and green lines indicate the error regions derived from a simulated observation $(50 \mathrm{ks}$ exposure time) with the Athena WFI and X-IFU instruments, respectively. Right panel: Simulated spectrum of $\mathrm{BD}+37^{\circ} 442$ for a 50 -ks long observation with the Athena/WFI instrument.

range $0.1-10 \mathrm{keV}$, would give a limit on $\dot{M}_{\mathrm{W}}$ in the range $(2-4) \times 10^{-13} M_{\odot}$ $\mathrm{yr}^{-1}$. This value is inconsistent with the theoretical predictions of Vink and Cassisi (2002) if CD -30 11223 has solar or higher abundances (see Fig. 1).

In Fig. 1 we show also the upper limits on $\dot{M}_{\mathrm{W}}$ obtained from the Xray observations of three sdB binaries which might have NS companions (Mereghetti et al., 2011a). These limits are quite constraining, but subject to the effective presence of a NS in these systems, which is based on the mass limits derived under the assumption that the subdwarf rotates synchronously. Further observations to confirm this are required. On the other hand, the current upper limits derived for the other sdB stars with proposed WD companions are two or three orders of magnitude higher and thus not particularly constraining for the theoretical models.

\section{Conclusions and future prospects}

The results obtained for the small, but rapidly growing, sample of hot subdwarfs detected in the X-ray range, show the potential of X-ray obser- 
vations in the study of these objects. Unfortunately, detailed analysis such as those carried out for several normal OB stars cannot be done for these relatively faint sources. For example, the element abundances of the thermal plasma models used to fit the sdO spectra could not be constrained by the X-ray data currently available. Therefore, the abundances had to be fixed to the values derived from optical studies, when available, or otherwise based on informed best guesses.

The increased sensitivity and spectral resolution of future X-ray telescopes will provide much better information on the spectral properties of the X-ray emission and lead to the discovery of many new sources. As an illustration of what could be obtained with Athena, the next large X-ray satellite approved by the European Space Agency for a launch in the late 2020s, we have simulated X-ray spectra of $\mathrm{BD}+37^{\circ} 442$ based on the expected performances of the WFI and X-IFU instruments (Rau, 2014, Barret et al., 2014). The analysis of these simulated data (see Fig. 6) indicate that it will be possible to obtain much more accurate measures of the plasma temperatures and constrain within $\sim 10 \%$ the abundances of $\mathrm{C}, \mathrm{N}, \mathrm{O}, \mathrm{Si}$ and $\mathrm{Fe}$, providing a measure independent of that derived from optical/UV analysis.

It is also important to increase the sample of hot subdwarfs detected in X-rays in order to consolidate the comparison with normal early type stars and to explore possible dependences on different parameters, such as surface gravity, temperature and composition. While future satellites will certainly allow us to reach fainter limiting fluxes, also the current facilities can in principle provide many new detections, since only a very small number of targets have been observed so far. One interesting question is whether isolated sdB stars are X-ray emitters.

More optical/UV studies of the X-ray emitting hot subdwarfs are needed to derive better estimates of their atmospheric and wind parameters. The accurate distances which will soon be provided by the Gaia mission will be crucial to reduce many of the uncertainties involved in these estimates. A multi-wavelength approach can be very effective to discover other systems such as HD 49798 and $\mathrm{CD}-30^{\circ} 11223$, in which the presence of a compact companion is certain. Besides their intrinsic interest from the point of view of binary evolution models, such systems will allow us to fully exploit the X-ray data to better characterize the poorly known winds of hot subdwarfs.

\section{Acknowledgements}

We thank the organizers of the Science Event "X-ray Astrophysics of Hot 
Massive Stars" at the 2014 COSPAR in Moscow and the participants to the Seventh Meeting on Hot Subdwarfs in Oxford for very interesting discussions. We are grateful to all our collaborators in the X-ray and optical observations of hot subdwarfs, and in particular to A.Tiengo, P.Esposito, G.L.Israel, U. Heber and S.Geier. This work has been partially supported from PRIN INAF 2014 .

\section{References}

\section{References}

Barret, D., den Herder, J., Piro, L., X-IFU Consortium, 2014. The X-ray Integral Field Unit of the Athena X-ray Observatory, in: The X-ray Universe 2014, p. 2.

Bauer, F., Husfeld, D., 1995. Metal abundances in subdwarf O stars. Astron. Astrophys. 300, 481.

Berghoefer, T.W., Schmitt, J.H.M.M., Cassinelli, J.P., 1996. The ROSAT allsky survey catalogue of optically bright OB-type stars. Astron. Astrophys. Suppl. 118, 481-494.

Bhattacharya, D., van den Heuvel, E.P.J., 1991. Formation and evolution of binary and millisecond radio pulsars. Physics Reports 203, 1-124. doi:10. 1016/0370-1573(91)90064-S.

Castor, J.I., Abbott, D.C., Klein, R.I., 1975. Radiation-driven winds in Of stars. Astrophys. J. 195, 157-174. doi:10.1086/153315.

Di Stefano, R., Voss, R., Claeys, J.S.W., 2011. Spin-up/Spin-down Models for Type Ia Supernovae. Astrophys. J. Lett. 738, L1. doi:10.1088/ 2041-8205/738/1/L1,

Geier, S., Heber, U., Podsiadlowski, P., Edelmann, H., Napiwotzki, R., Kupfer, T., Müller, S., 2010. Hot subdwarf stars in close-up view. I. Rotational properties of subdwarf B stars in close binary systems and nature of their unseen companions. Astron. Astrophys. 519, A25. doi:10.1051/0004-6361/201014465, 
Geier, S., et al., 2013. A progenitor binary and an ejected mass donor remnant of faint type Ia supernovae. Astron. Astrophys. 554, A54. doi:10.1051/ 0004-6361/201321395,

Hamann, W., Gruschinske, J., Kudritzki, R.P., Simon, K.P., 1981. Mass loss from O subdwarfs. Astron. Astrophys. 104, 249-255.

Hamann, W.R., 2010. Stellar winds from hot low-mass stars. Astrophysics and Space Science 329, 151-158. doi:10.1007/s10509-010-0344-8.

Harnden, Jr., F.R., Branduardi, G., Gorenstein, P., Grindlay, J., Rosner, R., Topka, K., Elvis, M., Pye, J.P., Vaiana, G.S., 1979. Discovery of an X-ray star association in VI Cygni /Cyg OB2/. Astrophys. J. Lett. 234, L51-L54. doi:10.1086/183107.

Heber, U., 2009. Hot Subdwarf Stars. ARA\&A 47, 211-251. doi:10.1146/ annurev-astro-082708-101836.

Heber, U., Geier, S., Irrgang, A., Schneider, D., Barbu-Barna, I., Mereghetti, S., La Palombara, N., 2014. The Mysterious sdO X-ray Binary BD $+37^{\circ} 442$, in: van Grootel, V., Green, E., Fontaine, G., Charpinet, S. (Eds.), 6th Meeting on Hot Subdwarf Stars and Related Objects, p. 307.

Heber, U., Maxted, P.F.L., Marsh, T.R., Knigge, C., Drew, J.E., 2003. Stellar Wind Signatures in sdB Stars?, in: Hubeny, I., Mihalas, D., Werner, K. (Eds.), Stellar Atmosphere Modeling, p. 251.

Hervé, A., Rauw, G., Nazé, Y., 2013. A detailed X-ray investigation of $\zeta$ Puppis. III. Spectral analysis of the whole RGS spectrum. Astron. Astrophys. 551, A83. doi:10.1051/0004-6361/201219734,

Hurley, J.R., Tout, C.A., Wickramasinghe, D.T., Ferrario, L., Kiel, P.D., 2010. Formation of binary millisecond pulsars by accretion-induced collapse of white dwarfs. MNRAS 402, 1437-1448. doi:10.1111/j. 1365-2966.2009.15988.x.

Husfeld, D., 1987. Non-LTE analyses of extremely helium-rich subluminous O stars, in: A. G. D. Philip, D. S. Hayes, \& J. W. Liebert (Ed.), IAU Colloq. 95: Second Conference on Faint Blue Stars, pp. 237-246. 
Iben, I.J., Tutukov, A.V., 1994. Helium-accreting degenerate dwarfs as presupernovae and scenarios for the ultrasoft x-ray sources. Astrophys. J. 431, 264-272. doi:10.1086/174484.

Israel, G.L., Stella, L., Angelini, L., White, N.E., Kallman, T.R., Giommi, P., Treves, A., 1997. The Discovery of 13 Second X-Ray Pulsations from the Hydrogen-depleted Subdwarf O6 Star Binary HD 49798. Astrophys. J. Lett. 474, L53. doi:10.1086/310418.

Jeffery, C.S., Hamann, W.R., 2010. Stellar winds and mass loss from extreme helium stars. MNRAS 404, 1698-1710. doi:10.1111/j.1365-2966.2010. 16410.x.

Krtička, J., Kubát, J., 2010. NLTE wind models of hot subdwarf stars. Astrophysics and Space Science 329, 145-150. doi:10.1007/ s10509-010-0385-z,

Kudritzki, R.P., Puls, J., 2000. Winds from Hot Stars. ARA\&A 38, 613-666. doi:10.1146/annurev.astro.38.1.613.

Kudritzki, R.P., Simon, K.P., 1978. Non-LTE analysis of subluminous O-star. The hydrogen-deficient subdwarf O-binary HD 49798. Astron. Astrophys. 70, 653-663.

La Palombara, N., Esposito, P., Mereghetti, S., Novara, G., Tiengo, A., 2015. Follow-up observations of X-ray emitting hot subdwarf stars: the He-rich sdO BD $+37^{\circ}$ 1977. Astron. Astrophys. 580, A56. doi:10.1051/ 0004-6361/201526141,

La Palombara, N., Esposito, P., Mereghetti, S., Tiengo, A., 2014. Three new X-ray emitting O-type subdwarf stars discovered with Chandra. Astron. Astrophys. 566, A4. doi:10.1051/0004-6361/201423571,

La Palombara, N., Mereghetti, S., Tiengo, A., Esposito, P., 2012. Discovery of a Compact Companion to the Hot Subdwarf Star BD $+37^{\circ} 442$. Astrophys. J. Lett. 750, L34. doi:10.1088/2041-8205/750/2/L34,

Latour, M., Fontaine, G., Chayer, P., Brassard, P., 2013. A Non-LTE Analysis of the Hot Subdwarf O Star BD+28 4211. I. The UV Spectrum. Astrophys. J. 773, 84. doi:10.1088/0004-637X/773/2/84, 
Latour, M., Fontaine, G., Green, E.M., Brassard, P., 2015. A non-LTE analysis of the hot subdwarf $\mathrm{O}$ star BD $+28^{\circ} 4211$. II. The optical spectrum. Astron. Astrophys. 579, A39. doi:10.1051/0004-6361/201525999,

Liu, D.D., Zhou, W.H., Wu, C.Y., Wang, B., 2015. Is the X-ray pulsating companion of HD 49798 a possible type Ia supernova progenitor? ArXiv e-prints arXiv:1504.01461.

Marcolino, W.L.F., Bouret, J.C., Martins, F., Hillier, D.J., Lanz, T., Escolano, C., 2009. Analysis of Galactic late-type O dwarfs: more constraints on the weak wind problem. Astron. Astrophys. 498, 837-852. doi:10.1051/0004-6361/200811289,

Martins, F., Schaerer, D., Hillier, D.J., Meynadier, F., Heydari-Malayeri, M., Walborn, N.R., 2005. On stars with weak winds: the Galactic case. Astron. Astrophys. 441, 735-762. doi:10.1051/0004-6361:20052927,

Massey, P., Gronwall, C., 1990. The Kitt Peak spectrophotometric standards - Extension to 1 micron. Astrophys. J. 358, 344-349. doi:10.1086/168991.

Mereghetti, S., Campana, S., Esposito, P., La Palombara, N., Tiengo, A., 2011a. Search for X-ray emission from subdwarf B stars with compact companion candidates. Astron. Astrophys. 536, A69. doi:10.1051/0004-6361/ 201118045,

Mereghetti, S., La Palombara, N., Esposito, P., Gastaldello, F., Tiengo, A., Heber, U., Geier, S., Wilms, J., 2014. Constraints on the winds of hot subdwarf stars from X-ray observations of two sdB binaries with compact companions: CD -30 11223 and PG 1232-136. MNRAS 441, 2684-2690. doi:10.1093/mnras/stu773,

Mereghetti, S., La Palombara, N., Tiengo, A., Pizzolato, F., Esposito, P., Woudt, P.A., Israel, G.L., Stella, L., 2011b. X-Ray and Optical Observations of the Unique Binary System HD 49798/RX J0648.0-4418. Astrophys. J. 737, 51-58. doi:10.1088/0004-637X/737/2/51,

Mereghetti, S., La Palombara, N., Tiengo, A., Sartore, N., Esposito, P., Israel, G.L., Stella, L., 2013. X-ray emission from the luminous O-type subdwarf HD 49798 and its compact companion. Astron. Astrophys. 553, A46. doi:10.1051/0004-6361/201321271, 
Mereghetti, S., Tiengo, A., Esposito, P., La Palombara, N., Israel, G.L., Stella, L., 2009. An Ultramassive, Fast-Spinning White Dwarf in a Peculiar Binary System. Science 325, 1222-1223. doi:10.1126/science.1176252.

Nazé, Y., 2009. Hot stars observed by XMM-Newton. I. The catalog and the properties of OB stars. Astron. Astrophys. 506, 1055-1064. doi:10.1051/ 0004-6361/200912659,

Nelemans, G., 2010. Population synthesis of Galactic subdwarf B stars. Astrophysics and Space Science 329, 25-31. doi:10.1007/ s10509-010-0392-0,

Owocki, S., 2013. Stellar Winds. p. $735 . \quad$ doi:10.1007/ 978-94-007-5615-115.

Pallavicini, R., Golub, L., Rosner, R., Vaiana, G.S., Ayres, T., Linsky, J.L., 1981. Relations among stellar X-ray emission observed from Einstein, stellar rotation and bolometric luminosity. Astrophys. J. 248, 279-290. doi:10.1086/159152.

Rau, A., 2014. The Wide Field Imager for the Athena X-ray Observatory, in: The X-ray Universe 2014, p. 167.

Rebeirot, E., 1966. Une etoile a grande vitesse riche EN helium ionise, "BD+37 442", NE presentant pas les raies de l'hydrogene. C.r.hebd. Seanc. Acad. Sci. Paris 262, 1105-1106.

Seward, F.D., Forman, W.R., Giacconi, R., Griffiths, R.E., Harnden, Jr., F.R., Jones, C., Pye, J.P., 1979. X-rays from Eta Carinae and the surrounding nebula. Astrophys. J. Lett. 234, L55-L58. doi:10.1086/183108.

Stickland, D.J., Lloyd, C., 1994. Spectroscopic binary orbits from ultraviolet radial velocities. Paper 14: HD 49798. The Observatory 114, 41-44.

Tauris, T.M., Sanyal, D., Yoon, S.C., Langer, N., 2013. Evolution towards and beyond accretion-induced collapse of massive white dwarfs and formation of millisecond pulsars. Astron. Astrophys. 558, A39. doi:10.1051/0004-6361/201321662,

Thackeray, A.D., 1970. The spectroscopic orbit of the O-type subdwarf HD 49798. MNRAS 150, 215-225. 
Thejll, P., Ulla, A., MacDonald, J., 1995. Infrared flux excesses from hot subdwarfs. Astron. Astrophys. 303, 773.

Unglaub, K., 2008. Mass-loss and diffusion in subdwarf B stars and hot white dwarfs: do weak winds exist? Astron. Astrophys. 486, 923-940. doi:10.1051/0004-6361:20078019,

Usov, V.V., 1988. Generation of Gamma-Rays by a Rotating Magnetic White Dwarf. Soviet Astronomy Letters 14, 258.

Vennes, S., Kawka, A., O'Toole, S.J., Németh, P., Burton, D., 2012. The Shortest Period sdB Plus White Dwarf Binary CD-30 11223 (GALEX J1411-3053). Astrophys. J. Lett. 759, L25. doi:10.1088/2041-8205/759/ 1/L25,

Vink, J.S., Cassisi, S., 2002. Hot horizontal branch stars: Predictions for mass loss. Winds, rotation, and the low gravity problem. Astron. Astrophys. 392, 553-562. doi:10.1051/0004-6361:20020917.

Vink, J.S., de Koter, A., Lamers, H.J.G.L.M., 2001. Mass-loss predictions for $\mathrm{O}$ and $\mathrm{B}$ stars as a function of metallicity. Astron. Astrophys. 369, 574-588. doi:10.1051/0004-6361:20010127,

Wang, B., Han, Z., 2010. A likely candidate of type Ia supernova progenitors: the X-ray pulsating companion of the hot subdwarf HD 49798. Research in Astronomy and Astrophysics 10, 681-688. doi:10.1088/1674-4527/10/ 7/007,

Wang, B., Han, Z., 2012. Progenitors of type Ia supernovae. New Astronomy Reviews 56, 122-141. doi:10.1016/j.newar.2012.04.001,

Werner, K., Dreizler, S., Haas, S., Heber, U., 1998. Iron and Nickel Abundances in DA White Dwarfs and SDO Stars, in: Wamsteker, W., Gonzalez Riestra, R., Harris, B. (Eds.), Ultraviolet Astrophysics Beyond the IUE Final Archive, p. 301. 